\title{
A New Approach in Reducing MEMS Inertial Gyro Random Errors Using Particle Filter
}

\author{
Yingjie $\mathrm{Hu}$
}

\begin{abstract}
The accuracy of MEMS inertial sensors is affected by random errors. Kalman Filter is the commonly used approach in reducing the random errors of MEMS sensor output. However, this approach is restricted by some dissatisfaction e.g. the divergent problem and the fixed noise covariance matrix $Q$ and $R$ 's inability to represent the dynamic noise characteristics of the system. In this paper, Particle filtering method is employed to reduce the random errors of MEMS gyro output. By a set of samples, Particle Filter is able to represent the posterior distribution of the states in a dynamic system when partial observations are made and random perturbations are present in sensor outputs. Experiments with artificial data sequence as well as authentic ADIS16445 inertial gyro output are conducted and then Particle Filter and Kalman Filter method are introduced to process these signals. Allan Variance analysis reveals that two main random errors are notably diminished after both filtering method and noticeably Particle Filter is superior to Kalman Filter in reducing MEMS gyro random noise.
\end{abstract}

Index Terms-MEMS gyro, random error, Particle Filter, Kalman Filter.

\section{INTRODUCTION}

Advances in MEMS(micro-electro-mechanical-system) techniques bring about the extensive application of MEMS sensors e.g. automobiles, smart phones, wearable devices and so forth. In the application of automobile navigation and positioning, the accuracy of MEMS inertial sensors influence the performance of INS(inertial navigation system) and thus the accuracy of vehicle positioning. The output of MEMS inertial sensors suffer from several types of errors which generally can be classified into two categories: deterministic error and random error [1]. In this paper, Focus is situated in the approaches to deal with random errors. Traditionally, the Allan variance is employed to identify the random error sources and corresponding noise parameters [2]. Based on prior knowledge of the random error sources, many methods have been explored to deal with them.

Normally, some digital filter techniques can be used to directly remove the random noise on specific frequency band, such as using a low pass filter to filter out the low frequency noise or using wavelet de-noising technology to remove the high frequency random noise [3]. However, this kind of methods may risk losing some useful information of the signal due to improper choice of wavelet level of decomposition. Apart from these digital filters that directly remove the random noise, Kalman Filter is a popular method and it enjoys

Manuscript received March 2, 2019; revised June 30, 2019.

Yingjie Hu is with the School of Automotive Studies, Tongji University, Shanghai 201804 China (e-mail: redheart9527@163.com). many applications in the context of vehicle navigation and positioning such as the initial alignment process of SINS [4] and the sensor fusion of integrated navigation system [5]. In order to eliminate the random error of MEMS inertial gyro, Kalman Filter is combined with time series analysis. First based on time series analysis, an ARMA(autoregressive-moving-average) model is built and from it Kalman Filter state equation and measurement equation are constructed to filter the random noise in an iterative manner [6]. The conventional Kalman Filter entails fixed process noise and measurement noise covariance matrix $\mathrm{Q}$ and $\mathrm{R}$. The prior knowledge of noise statistics is not enough to represent process noise covariance matrix of a dynamic system precisely. Thus fixed noise covariance can lead to divergent problems [7]. Apart from Kalman Filter methods, SVM(support vector machine) [8] and neural network method [9] are also used to deal with the random errors of MEMS inertial sensors. But these methods are flawed by slow training convergence speed, local minima and over-fitting problems.

Over the past decades, a series of nonlinear filtering methods based on Bayesian approach have been introduced to solve problems in tracking maneuvering target, perception and navigation, signal compression and transmission and so forth [10]. These sequential Monte Carlo approaches include Bootstrap Filter, Condensation Tracks, Particle Filter and Interacting Particle Approximations etc. [11]. All of them are based on the sequential importance sampling(SIS) algorithm.

By a set of random state samples, the objective of Particle Filter is to represent the posterior distribution of the states in dynamical systems when partial observations are made and random perturbations are present in the sensors as well as in the dynamical systems. To put it mathematically, the posterior probability distribution at epoch $\mathrm{k}-1$ is denoted as $p\left(x_{k-1} \mid z_{1: k-1}\right)$, samples drawn from the posterior distribution are called particles. Through state update and measurement update, the posterior probability of these $\mathrm{n}$ samples approximates the system posterior probability distribution $p\left(x_{k} \mid z_{1: k}\right)$. Just like all other Bayesian filtering algorithm, Particle Filter constructs recursively the posterior probability distribution from $p\left(x_{k-1} \mid z_{1: k-1}\right)$ one time step earlier. Particle Filter is nonparametric and therefore can represent a much broader spectrum of distribution. Another advantage is its ability to model nonlinear transformations of random variables [12].

In this paper, we introduce Particle Filter in the MEMS inertial signal processing and compare the effect of reducing random errors of gyro output between Kalman Filter and Particle Filter. In Section II a basic description of Particle Filter and Kalman Filter algorithm is given. Afterwards in 
Section III, time series analysis is used to build $\operatorname{ARMA}(\mathrm{p}, \mathrm{q})$ model of the gyro signal, which leads to the state equation and measurement equation of the system. And then the Kalman Filter and Particle Filter are implemented to eliminate the random error of gyro signal respectively, the filtering results for both approaches are compared through Allan variance analysis. Finally, a conclusion is drawn in Section IV.

\section{Two FILTERING AlgORITHM}

\section{A. Particle Filter}

To describe a dynamical system, the state space equations are shown in (1):

$$
\begin{aligned}
& x_{k}=f_{k}\left(x_{k-1}, w_{k-1}\right) \\
& z_{k}=h_{k}\left(x_{k}, v_{k}\right)
\end{aligned}
$$

where, $\quad f_{k}: R^{n_{x}} \times R^{n_{w}} \rightarrow R^{n_{x}} \quad, \quad h_{k}: R^{n_{x}} \times R^{n_{v}} \rightarrow R^{n_{z}} \quad$ are possibly nonlinear function. $w_{k}, v_{k}$ are process noise sequence and measurement noise sequence respectively and they are independent. $n_{x}, n_{w}$ are the dimensions of the state vector and process noise while $n_{z}, n_{v}$ are the dimensions of the measurement vector and measurement noise.

The main idea of Particle Filter is to approximate the posterior distribution $p\left(x_{k} \mid z_{1: k}\right)$ by a set of random state samples drawn this posterior. These particles can be denoted: $\chi_{t}=x_{t}^{[1]}, x_{t}^{[2]}, \ldots, x_{t}^{[M]}$. Corresponding to each particle, weights $\omega_{t}^{[m]}$ are defined to evaluate how well they predict the current measurement. The particles with large weight are kept and those with small weight are thrown away. This step is called resampling. The resampling algorithm draws with replacement $M$ particles from the particle set $\chi_{t}=x_{t}^{[1]}, x_{t}^{[2]}, \ldots, x_{t}^{[M]}$. The probability of drawing each particle is given by its weight. The resampling step transforms the particle set of $\mathrm{M}$ particles into another particle set of the same size. The resulting sample set usually possesses many duplicates, since particles are drawn with replacement. And after resampling, they are distributed approximately according to the posterior distribution $p\left(x_{k} \mid z_{1: k}\right)$.The basic algorithm for Particle Filter is shown using pseudo-code as below:

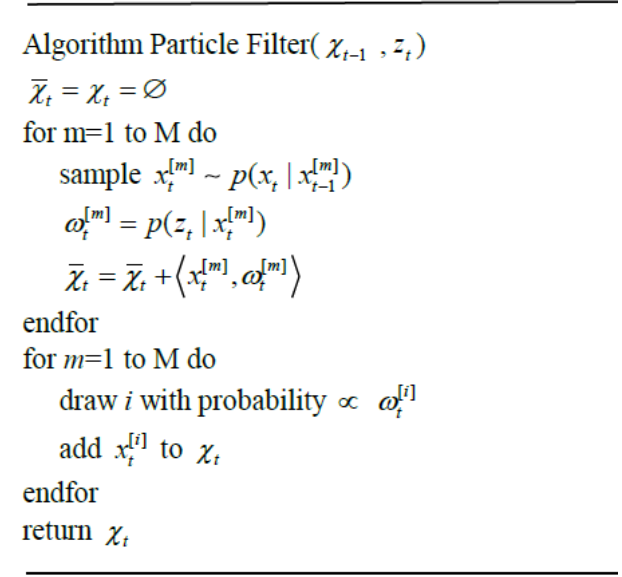

\section{B. Kalman Filter}

Kalman Filter is an algorithm that uses a series of measurements observed over time, containing statistical noise and other inaccuracies, and produces estimates of unknown variables that tend to be more accurate than those based on one single measurement alone. Among its diverse applications, in this case, Kalman Filter forms a contrast with Particle Filter mentioned above in reducing the random errors of MEMS inertial gyro outputs.

To design a standard Kalman Filter, the system state equation and measurement equation are initially determined in (2):

$$
\left\{\begin{array}{c}
x_{k}=A x_{k-1}+w_{k-1} \\
z_{k}=H x_{k}+v_{k}
\end{array}\right.
$$

where, $x_{k}$ denotes state vector at epoch $\mathrm{k} ; z_{k}$ denotes measurement vector at epoch $\mathrm{k}$; $\mathrm{A}$ is the state transition matrix; $\mathrm{H}$ is the observation matrix; $w_{k-1}, v_{k}$ represents the process noise and measurement noise respectively. One prerequisite of standard Kalman Filter is that $w_{k-1}, v_{k}$ are white Gaussian noise with zero mean and independent from each other. The noise item $w_{k-1}, v_{k}$ have covariance matrices $Q$ and $R$ respectively.

The procedures of a standard Kalman Filter are expressed in the following steps:

(1) step prediction of the state:

$$
x_{k \mid k-1}=A x_{k-1}
$$

(2) step prediction of the error covariance matrix:

$$
P_{k \mid k-1}=A P_{k-1} A^{T}+Q
$$

(3) calculate Kalman gain:

$$
K_{k}=P_{k \mid k-1} H^{T}\left[H P_{k \mid k-1} H^{T}+R\right]^{-1}
$$

(4) estimate of the state:

$$
x_{k}=x_{k \mid k-1}+K_{k}\left(z_{k}-H x_{k \mid k-1}\right)
$$

(5) estimate of the error covariance matrix:

$$
P_{k}=\left(I-K_{k} H\right) P_{k \mid k-1}
$$

The initial state and error covariance matrix are $\hat{x}_{0}$ and $\hat{P}_{0}$. Given $\hat{x}_{0} \hat{P}_{0},(3) \sim(7)$ are calculated circularly to obtain the optimal estimation of the state.

\section{EXPERIMENT AND ANALYSIS}

\section{A. Experimental Settings}

The MEMS inertial sensor involved in the experiment is ADIS16445 produced by Analog Devices. A triaxial gyroscope and a triaxial accelerometer are packaged in a module and have a standard connector interface for data transmission. Data acquisition device is INCA software connected with ADIS16445 with ValueCAN. The experiment is conducted at room temperature to preclude the influence of temperature. Sampling frequency is $100 \mathrm{~Hz}$ and 0.5 -hour 
static data is collected for analysis. The test layout of the experiment is shown in Fig. 1.

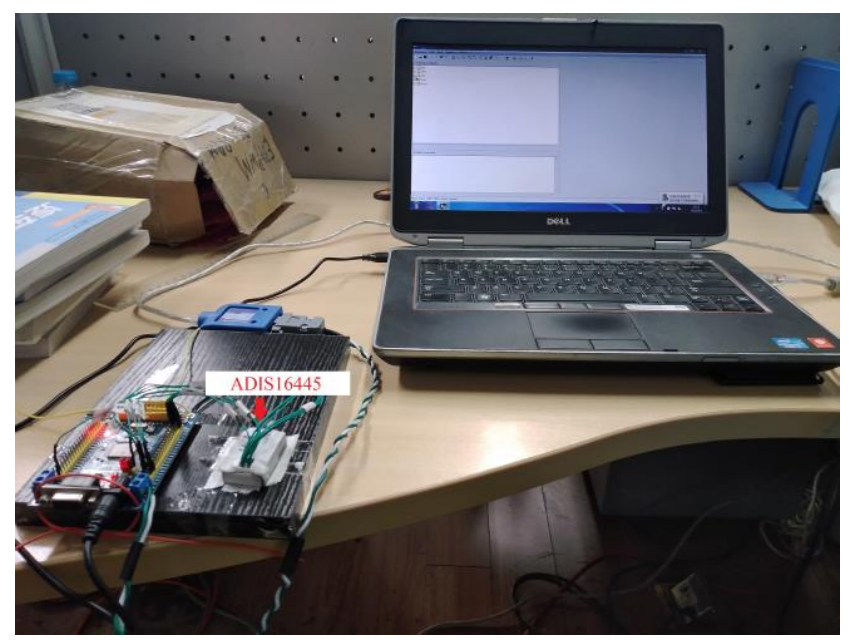

Fig. 1. Experimental layout.

Raw data collected for a length of 0.5 hour is at first preprocessed to remove the abnormal points according to $3 \sigma$ principles and then subtracting bias from the original signal. After this preprocessing, Z-axis angle rate of ADIS16445 gyro is plotted in Fig. 2 (all signal mentioned in the rest of this paper is based on the data of Z-axis gyro).

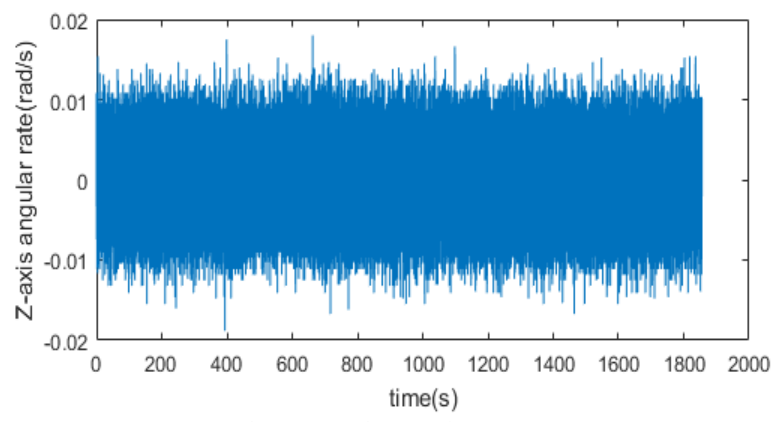

Fig. 2. Z-axis angular rate.

\section{B. Time Series Analysis}

In order to introduce Kalman Filter or Particle Filter, state space model of gyro output should be determined at first. Here time series analysis is used to build ARMA model of stationary, normally distributed and zero-mean signal.

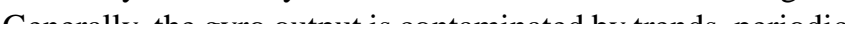

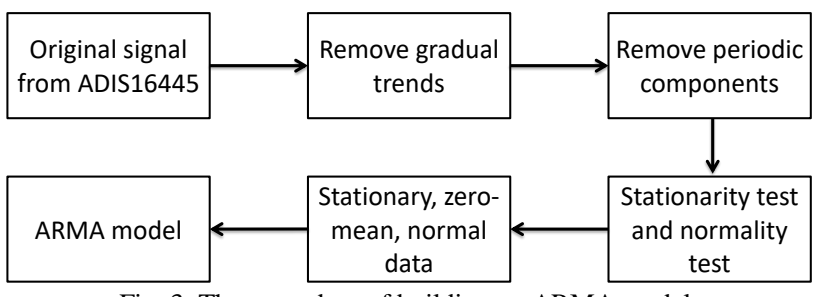

Fig. 3. The procedure of building an ARMA model.

1) At first it is necessary to extract the gradual trends from the raw data. Normally the trend errors are characterized by polynomials or exponential functions. The proper selection of fitting function and its order is based on 2 dimensional scatter plots and empirical experience.

2) Periodic test is conducted on the signal form last step to ensure that the signal is free of periodic components. If there is a periodic component with the period $\mathrm{D}$, then D-order difference method is an effective way to get rid of the corresponding periodic component [15].

3) Stationarity test and normality test are conducted on the signal form last step to make sure the signal is stationary and normally distributed, which is an important premise for ARMA model building.

4) After the processing of the signal, ARMA model can be established as (8).

$$
\begin{aligned}
x(n)= & a_{1} x(n-1)+a_{2} x(n-2)+\cdots \\
& +a_{p} x(n-p)+w(n)+b_{1} w(n-1) \\
& +b_{2} w(n-2)+\cdots+b_{q} w(n-q) \\
= & \sum_{k=1}^{p} a_{k} x(n-k)+\sum_{k=0}^{q} b_{k} w(n-k)
\end{aligned}
$$

where, $x(n)$ is signal value at epoch $n, w$ is white noise, $w \sim\left(0, \sigma^{2}\right) . p, q$ are the order of $\operatorname{ARMA}(p, q)$. (8) shows that the random variable at epoch $\mathrm{n}$ is correlated with its previous values within $\mathrm{p}$ steps, white noise and the previous values of white noise within $q$ steps. When $q=0$ or $p=0, \operatorname{ARMA}(p, q)$ converts into $\operatorname{AR}(p)$ or $\operatorname{AR}(q)$.

Generally, $\operatorname{AR}(p)$ model is most frequently used to characterize the signal of MEMS gyro. Thus here $\operatorname{ARMA}(p, q)$ model is simplified as $\operatorname{AR}(p)$ model. According to truncation property of $\mathrm{PACF}$ (partial autocorrelation function) shown in Fig. 4. The order for $\operatorname{AR}(p)$ can be determined as 1 .

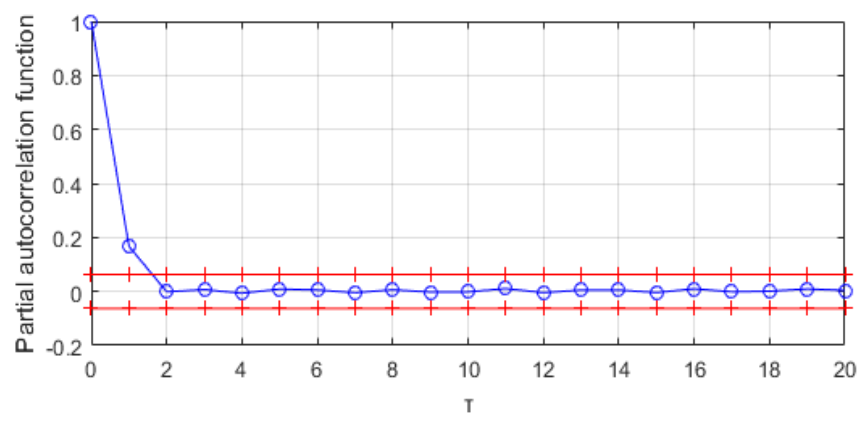

Fig. 4. Partial autocorrelation function.

Therefore, the model used to fit the output signal of ADIS16445 gyro can be determined and is expressed in (9).

$$
x(n)=0.174 x(n-1)+w(n)
$$

\section{Particle Filter and Kalman Filter}

After the AR(1) model is built, the system state equation and measurement equation can be expressed in (10)

$$
\begin{aligned}
& X(k)=A X(k-1)+w(k-1) \\
& Z(k)=H X(k)+v(k)
\end{aligned}
$$

where, $A=0.174, H=1, w(k-1), v(k)$ denotes process noise and measurement noise respectively, which are both Gaussian white noise with zero mean and independent from each other.

Initially, we build an artificial data sequence in conformity 
with the obtained AR(1) model and implement Particle Filter and Kalman Filter respectively on this artificial sequence to check out the estimation effects for both methods and then the authentic data collected from ADIS16445 gyro is processed using Particle Filter and Kalman Filter for comparison. It is expected the results will reveal a prevailing effect for Particle Filter over Kalman Filter in eliminating the random noise.

By means of Matlab, 100 points are created in conformity with $\mathrm{AR}(1): x(n)=0.174 x(n-1)+w(n)$. Particle Filter algorithm along with Kalman Filter algorithm are tested to estimate the state. with regard to the same data sequence, the filtering results are exhibited in Fig. 5, Fig. 6 and Fig. 7.

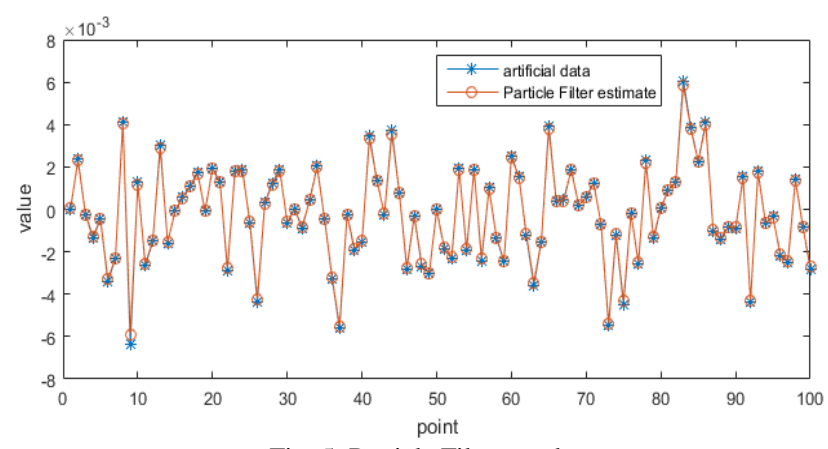

Fig. 5. Particle Filter results.

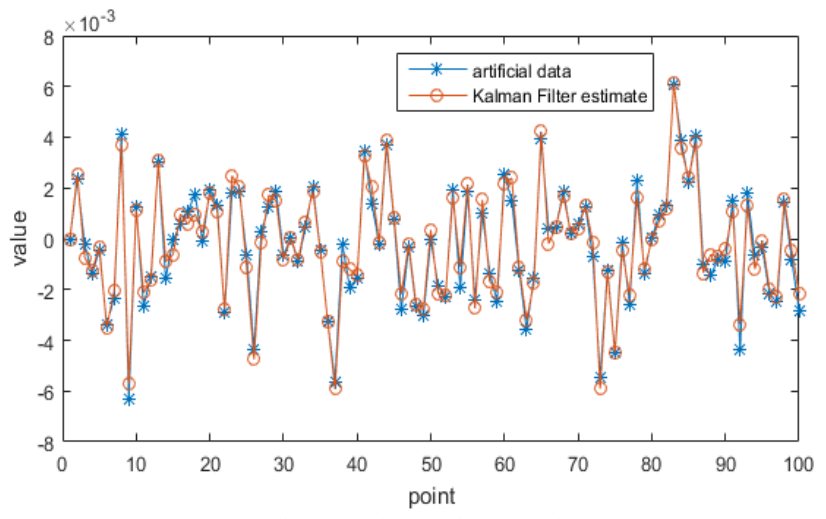

Fig. 6. Kalman Filter results.

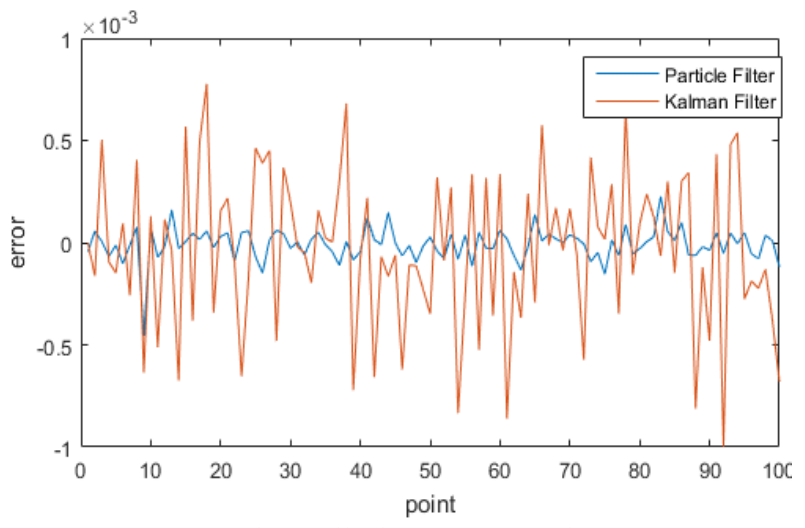

Fig. 7. Filtering error comparison.

Simulating results show that Particle Filter has a better effect in estimating the artificial data sequence than Kalman Filter. Evidently, the estimate errors of Particle Filter algorithm are less than those of Kalman Filter as are shown in Fig. 7. Next, both filtering algorithms are used to process the data collected from ADIS16445 gyro. Expectedly, the Particle Filter should have a better performance in dealing with the random errors than Kalman Filter. Test results are shown in Fig. 8, which proves that the Particle Filter is more effective in eliminating the random errors than Kalman Filter in application of MEMS inertial gyro ADIS16445.

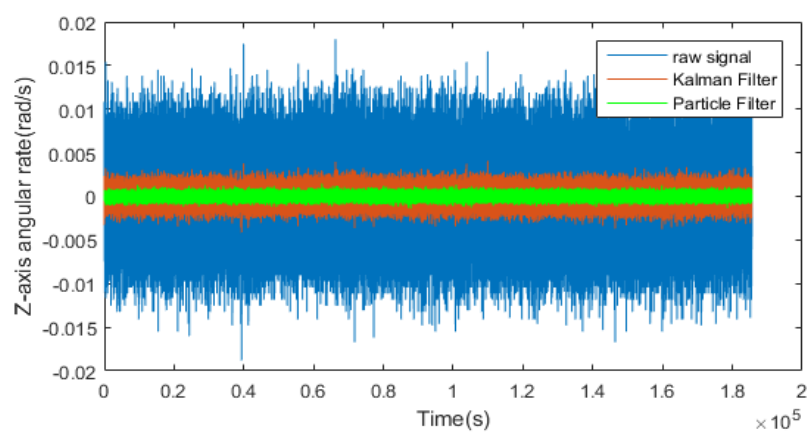

Fig. 8. The filtering contrast between the two filtering methods

Additionally, Allan variance method is also employed to analyze the effect of these filtering means quantitatively. The advantages of Allan variance lies in its capability to identify the specific random noise terms and corresponding characteristic parameters. Fig. 9 shows the Allan variance double logarithmic graphs of raw signal and the processed signal after two filtering methods respectively. Table I reveals the random noise parameters derived from Allan variance double logarithmic graphs for these three cases. According to the theory of Allan variance, two main random error sources can be identified according to the slopes of Allan variance graphs: angle random walk (slope $-1 / 2$ ), bias (slope 0 ). The error for Allan variance algorithm increases as the cluster time accumulates. Thus the right part of these curves which correspond to the slope $1 / 2$ embodies considerable errors and are not taken into consideration when identifying the random error sources. Then the parameters for these random error sources are calculated and listed in Table I Notably, after both filtering method, the random error parameters are significantly decreased. And after Particle Filter, these parameters are diminished greater compared with those after Kalman Filter method, e.g. Bias instability decreased in Kalman Filter case from $16.726^{\circ} / \mathrm{hr}$ to $4.138^{\circ} / \mathrm{hr}$ and in Particle Filter case form $16.726^{\circ} / \mathrm{hr}$ to $0.744^{\circ} / \mathrm{hr}$.

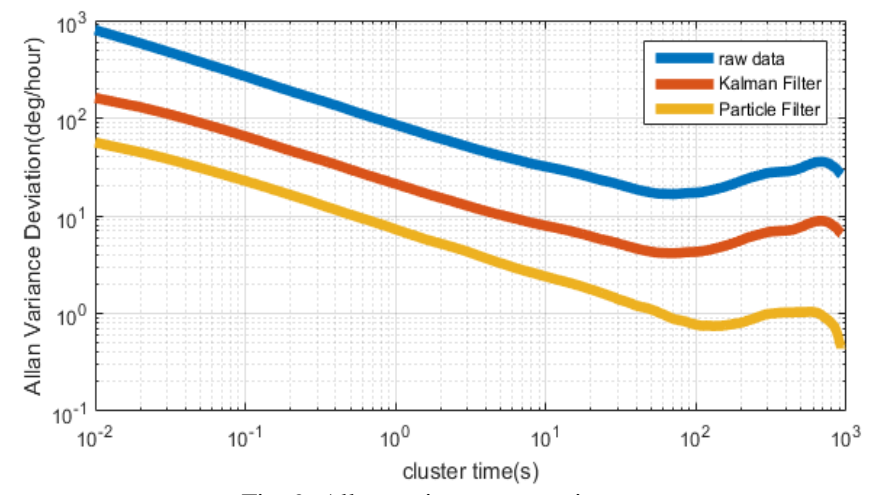

Fig. 9. Allan variance comparison.

TABLE I: RANDOM ERROR PARAMETERS

\begin{tabular}{lll}
\hline \hline & $\begin{array}{l}\text { Angle Random Walk } \\
(\circ / \sqrt{h r})\end{array}$ & Bias Instability( $\left.{ }^{\circ} / h r\right)$ \\
\hline Raw Data & 1.426 & 16.726 \\
Kalman Filter & 0.353 & 4.138 \\
Particle Filter & 0.121 & 0.744 \\
\hline \hline
\end{tabular}




\section{CONCLUSION}

The accuracy of MEMS inertial gyro is negatively impacted by stochastic errors. This paper proposes a Particle Filtering method to eliminate the MEMS gyro random errors. First time series analysis is employed to establish ARMA model, from which the system state equations can be determined. Then Particle Filter method are introduced to eliminate the random errors of gyro signal and Kalman Filter method is also implemented in order to form contrast. Experiments using simulating data sequence as well as authentic ADIS16445 gyro output prove that the Particle Filter is an effective manner in suppressing the IMU gyro random noise and is more efficacious than Kalman Filter in this case. Allan variance analysis indicates that after Particle Filter, random error parameters are diminished greater compared with those after Kalman Filter method, e.g. Bias instability decreased in Kalman Filter case from $16.726 \% / \mathrm{hr}$ to $4.138 \% \mathrm{hr}$ and in Particle Filter case from $16.726^{\circ} / \mathrm{hr}$ to $0.744 \% / \mathrm{hr}$.

\section{REFERENCES}

[1] D. Unsal and K. Demirbas, "Estimation of deterministic and stochastic IMU error parameters," in Proc. Position Location and Navigation Symposium, 2012, pp. 862-868.

[2] N. El-Sheimy, H. Hou, and X. Niu, "Analysis and modeling of inertial sensors using Allan variance," IEEE Trans. on Instrumentation \& Measurement, 2007, pp. 140-149.

[3] S. Nassar and N. El-Sheimy, "Wavelet analysis for imroving INS and INS/DGPS navigation accuracy," Journal of Navigation, 2005, pp. 119-134.

[4] J. C. Fang and D. J. Wan, "A fast initial alignment method for strapdown inertial navigation system on stationary base," Journal of Southeast University, 1996, pp. 1501-1504.

[5] M. Ilyas, Y. Yang, Q. S. Qian, and R. Zhang, "Low-cost IMU/odometer/GPS integrated navigation aided with two antennae heading measurement for land vehicle application," in Proc. Control and Decision Conference, 2013, pp. 4521-4526.

[6] X.G. Ruan and M. M. Yu, "Modeling Research of MEMS Gyro Drift Based on Kalman Filter," in Proc. Control and Decision Conference, 2014, pp. 2949-2952.

[7] M. Narasimhappa, S. L. Sabat, P. Rangababu, and J. Nayak, “An improved adaptive kalman filter for denoising fiber optic gyro drift signal," in Proc. India Conference IEEE, 2014, pp. 1-6.

[8] G. Xu and X. Meng, "The MEMS IMU error modeling analysis using support vector machines," in Proc. International Symposium on Knowledge Acquisition and Modeling, 2009, pp. 335-337.

[9] X. Chen, "Modeling random gyro drift by time series neural networks and by traditional method," in Proc. International Conference on Neural Networks and Signal Processing, 2003, pp. 810-813.

[10] N. Bergman, "Recursive Bayesian estimation: Navigation and tracking applications," Linkoeping University, 1999, pp. 305-314.

[11] H. V. Trees and K. Bell, A Tutorial on Particle Filter for Online Nonlinear/NonGaussian Bayesian Tracking, Chichester, U.K.: Wiley, 2007, pp. 723-737.

[12] S. Thrun, Probabilistic Robotics, Cambridge, MA: MIT press, 2005, pp. 52-57.

[13] S. N. Maceachern, M. Clyde, and J. S. Liu, "Sequential importance sampling for nonparametric Bayes models: The Next Generation," Canadian Journal of Statistics, 1999, pp. 251-267.

[14] Y. Guo, F. Han, S. Du, G. Ma, and L. Zhu, "Performance analysis of MEMS gyro and improvement using kalman filter," in Proc. Control Conference IEEE, 2015, pp. 4789-4794.

[15] G. Yan, S. Li, and Y. Qin, Inertial Instrument Test and Data Analysis, Beijing, P.R.C.: National Defense Industry Press, 2012, pp. 92-94.

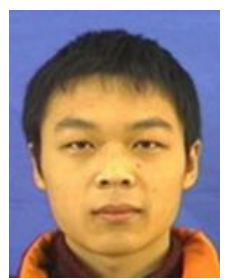

Yingjie Hu received the B.Eng. degree in automobile engineering from Tongji University, Shanghai, China, in 2017.

$\mathrm{He}$ is currently pursuing his master degree in automobile engineering in Tongji University, Shanghai, China. His research interests include MEMS sensor errors and vehicle navigation and positioning. 